Journal of Computer Science 2 (5): 455-459, 2006

ISSN 1549-3636

(C) 2006 Science Publications

\title{
TCP Optimal Performance in Wireless Networks Applications
}

\author{
${ }^{2}$ Emad Qaddoura, ${ }^{1}$ Abdelghani Daraiseh, ${ }^{3}$ Wesam Al Mobaideen, ${ }^{1}$ Rushdi Muammar and ${ }^{1}$ Soliman Al- \\ Walaie \\ ${ }^{1}$ Communications \& Computer Networks Unit, P\&CSD, Saudi Aramco \\ P.O. Box 10908, Dhahran, 31311 Saudi Arabia \\ ${ }^{2}$ Faculty of Computer Science and Information Technology, Applied Science University \\ Amman, 11931 Jordan \\ ${ }^{3}$ King Abdullah II School for Information Technology, The University of Jordan, Amman 11942 Jordan
}

\begin{abstract}
Mobile Ad Hoc Network (MANET) consists of dynamic topology as nodes in the network are mobile, and connected via wireless links. The nodes are grouped in clusters for the purpose of routing. Nodes within the cluster communicate directly. However, nodes communicate outside the cluster through a centralized node that is called a clusterhead. An elected clusterhead is assigned for communication with all other clusters. The centralized clusterhead can become a bottleneck and possibly cause a lower throughput for the clustering system. We propose a mechanism in which communication outside the cluster is distributed through separate clusterheads. We prove that the overall averaged throughput for the clustering system increases considerably, about doubles for our distributed number of clusterheads. We also show that the increase in overall averaged throughput is reduced by the increase in the overall averaged error rate ratio resulting from the added clusterheads. Additionally, the routing reliability to outside the cluster is increased since routes to different clusters use distinct clusterhead.
\end{abstract}

Key words: $\quad$ TCP optimal performance, Wireless and Wireline Networks Convergence

\section{INTRODUCTION}

Currently, most data applications on wireline networks use TCP/IP as an end-to-end flow and congestion avoidance protocol. $\mathrm{TCP}^{[5-7]}$ provides excellent performance for data transfer in networks with stationary users when congestion is mainly due to packet loss and unusual long delays ${ }^{[12]}$. For wireless or wireline networks, standard TCP responds to congestion by dropping transmission window size to a minimum, and exponentially backing off its retransmission timer. This technique lowers the utilization of the intermediate links in the networks and thereby controls congestion and long delays. Standard TCP suffers from throughput degradations in pure wireless networks, because it reacts to packet loss due to RF link fading as congestion. Fading occurs frequently in wireless networks, the throughput per user and the end-to-end network throughput therefore are unacceptable when standard TCP is employed. Several modified TCP protocols have been proposed to reduce the effect of RF related packet loss on TCP performance ${ }^{[1-9,11,13-14]}$.

The support of voice and data applications over networks comprising of an integrated wireline with wireless users is of an increasing importance in future technologies of Unified Networks. The Unified Networks refer to an integrated wireless and wireline networks with wireline, wireless, mobile and satellite users. While the proposed techniques in ${ }^{[2]}$ and ${ }^{[13]}$ have proven to be useful for pure wireless networks, they will not perform well in networks consisting of wireless and wireline users. As a result of being designed for wireline networks, standard TCP does not have the side information to distinguish between packet loss due to congestion and packet loss due to RF fading. Furthermore, the standard TCP does not have the capabilities to utilize the side information. The main contribution of this study is the method to obtain the maximum achievable throughput for an integrated wireless and wireline networks given today's TCPReno protocol and given its congestion control reliability. The maximum achievable throughput is obtained for heavily loaded network. The sensitivity of the system's parameters on the throughput will be highlighted. Any suggested modified TCP transport protocol or new transport protocol for Unified Networks should aim at approaching or exceeding this throughput. In effect, the performance of any modified TCP should be measured in terms of how close it approaches the maximum achievable throughput for integrated wireline and wireless networks.

The purpose of this study is to find the maximum achievable throughput for an integrated wireless and wireline network (Fig. 1, network-A) as a function of the number of wireless and wireline sessions and RF fading characteristics. It is understood that network-A in Fig. 1 must support the standard TCP transport

Corresponding Author: Emad Qaddoura, Faculty of Computer Science and Information Technology, Applied Science University, Amman, 11931 Jordan, Tel: +962-6-560-9999 
protocol without modifications. The approach for finding the maximum achievable throughput for network-A follows the following heuristic: maximum achievable throughput for network-A is obtained when the packet loss due to RF link is filtered out, but the characteristics of wireless traffic after filtering are correctly modeled and transported to network-A. Indirect TCP (I-TCP) ${ }^{[1]}$, is used for analysis purposes (used as the filtering mechanism at wireless LAN). Standard TCP Reno ${ }^{[7,15-16]}$ continues to be the transport protocol for network-A which is our wireline side of the network. Thus, the maximum achievable throughput is the throughput for network-A when "filtered" wireless packets and regular wireline packets are transported into network-A, congestion avoidance reliability remains unchanged.

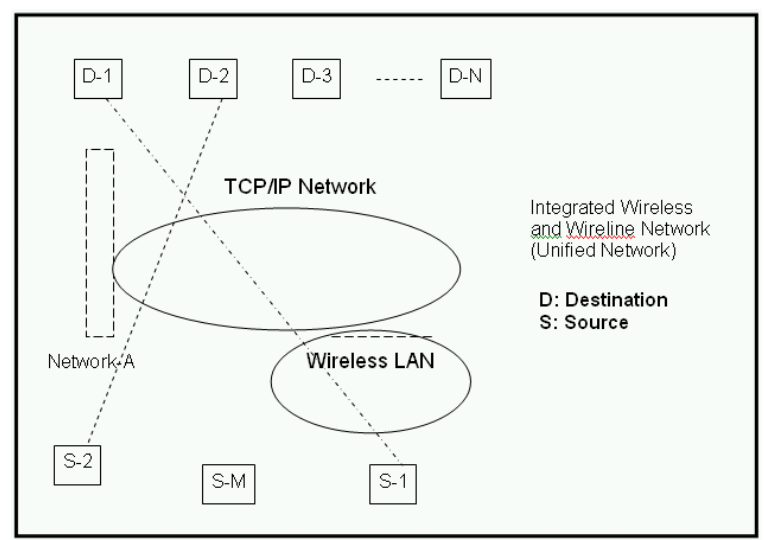

Fig. 1: Topology of integrated wireless/wireline network with I-TCP between S-1 and D-1.

Related work: Recent studies have focused on the poor performance of the TCP over wireless connections. Compared to wired networks, wireless networks have very low bit error rate, have more delayed packets, and less channel reliability ${ }^{[2,9]}$. Over wireless networks, and due to the high bit error rate, packets may get corrupted and lost considerably. Traditional TCP, when used over wireless networks, incorrectly interprets packet loss as a sign of congestion, backs off from further transmission, and reduces its congestion window. This consequently leads to degradation in the overall connection throughput ${ }^{[2,18-19]}$.

The MAC sub-layer may affect the behavior of TCP and its congestion control mechanism such as fast retransmit mechanism, which triggers the retransmission of dropped packets at a faster rate than the regular timeout mechanism requires ${ }^{[20-21]}$. Fast retransmit mechanism can not replace regular timeout TCP timers; it could only enhance it by speeding up the retransmission of a lost packet ${ }^{[22]}$. In other words, when the sender receives a certain number of duplicate ACKs of the same packet, it triggers the fast retransmit mechanism to retransmit the specified packet probably before the TCP sender times out ${ }^{[23]}$.
Different approaches to improve TCP over wireless links are classified into three basic groups, based on their fundamental philosophy: end-to-end solutions, split-connection solutions and link-layer solutions ${ }^{[3]}$. Indirect-TCP $^{[1]}$ is a split-connection solution that uses standard TCP for its connection over the wireless link. I-TCP improves TCP performance over wireless links, deals with high BER, and hides handoffs from the fixed host and transfers the connection state from the base station which is currently servicing the mobile host to the new base station which is currently serving it. On the other hand, I-TCP does not maintain end-to-end semantics. M-TCP ${ }^{[17]}$ is another split connection protocol proposed for cellular networks and mobile hosts to cope with frequent disconnections and handoffs. On contrast to I-TCP, M-TCP does maintain end-to-end semantics. M-TCP doesn't mainly cope with high bit error rate problems and leaves it for link layer solutions. Our simulated framework is out lined in Fig. 2 below, where the connection between the fixed host and the base station uses standard TCP. However, the TCP over the wireless channel is based on our developed wireless based TCP. Therefore, there is no end-to-end TCP connection between the FH and MH.

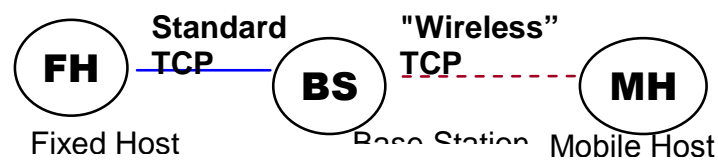

Fig. 2: Split Connection Approach

\section{BACKGROUND}

An overview of TCP's slow start, congestion avoidance, fast retransmit, retransmission timer backoff and RTT estimation is presented in this section. This section also provides the background information needed in the simulation model and subsequent sections. In addition, the important information necessary for understanding the numerical results is included.

In standard TCP, congestion is started as a result of timeout. Two of the main variables that are used to manage the behavior of the growth for the transmission window in TCP are Slow Start Threshold (SSTHRESH) and Congestion WiNDow (CWND). Initially, TCP starts in the slow start phase, $(\mathrm{CWND}=1$, SSTHRESH $=64 \mathrm{~K})$, in which the transmission window (CWND) grows exponentially as long as the CWND is less than or equals to SSTHRESH. Once the value of CWND is greater than SSTHRESH, the congestion avoidance phase is entered, and the CWND window grows linearly. Slow start is entered in the beginning of a TCP session, and again as a result of congestion. A timeout causes TCP to re-enter the slow start phase, in which the setting of the CWND and SSTHREH variables differs among TCP implementations. In TCP Tahoe, the SSTHRESH is set to half of the CNWD, and the 
CWND to the size of one TCP segment. In TCP Reno, the CWND is set to half of its current value.

The congestion window grows exponentially with the arrival of acknowledgements, until SSTHRES becomes less than the CWND. This triggers congestion avoidance phase and indicates a linear growth in the congestion window. The linear growth in the CWND is accomplished by incrementing the CWND by one segment for every full window's worth of acknowledged segments. The retransmission timer follows an exponential backoff technique. Once the retransmission expires, it is restarted with a value that is twice the value of its current timer. A timeout event occurs when the retransmission timer counts down to zero.

Fast retransmit allows TCP to retransmit lost packets without waiting for the retransmission timer to expire. Three duplicate acknowledgements are interpreted to indicate a loss of the segment. In this case, SSTHRESH is set to half of the minimum of the CWND and the receiver's advertised window. The CWND is set to SSTHRESH plus 3 times the segment size. Each time a duplicate acknowledgement is received, CWND is incremented by the size of one segment. Fast recovery allows TCP to start congestion avoidance, instead of slow start, after fast retransmission allows the CWND to be larger that SSTHRESH. When the first received acknowledgement for a new data segment is received, CWND is set to the value of SSTHRESH. This activates the congestion avoidance algorithm.

Indirect-TCP (I-TCP) ${ }^{[1]}$ is a transport layer protocol for mobile hosts, that is based on Indirect protocol model. I-TCP is fully compatible with TCP/IP on the fixed network and built around the following simple concepts: (1) A transport layer connection between a Mobile Host (MH) and a Fixed Host (FH) is established as two separate connections - one over the wireless medium and the other over the fixed network with the current Mobile Support Routers (MSRs) as the center point. (2) If the MH switches cells during the lifetime of an I-TCP connection, the center point of the connection moves to the new MSR. (3) The FH is completely unaware of the indirection and is not affected even when the MH switches cells, and when the center point of the I-TCP connection moves from one MSR to another. Since I-TCP protocols maintain a significant amount of state at the base station per TCP connection, handoff procedures can be complicated and slow, because of packet re-ordering during and after a handoff. The intention of the I-TCP is twofold: (i) shield the wireline part from the lossy nature of the wireless side, and (ii) hide handoffs form the fixed host and transfer the connection from the base station which is currently servicing the mobile host to the new base station which is receiving it.

System model and analysis: The traffic model for wireless and wireline FTP simulations considers the following: sessions are first discussed here.
- U number of FTP sessions over network-A, where both connection ends are at the wireline network.

- $\mathrm{U} 1$ of these sessions are wireline sessions, both end of the connection are wireline.

- $\mathrm{U} 2$ are wireless sessions where one end of the connection at the wireless side of the network, and the other end is at the wireline side, (U-U1).

The traffic associated with U2 sessions encounters two transport protocols:

- The first transport protocol is the WLAN transport protocol can be any suggested protocol for pure wireless LAN;

- The second transport protocol is the standard TCPReno protocol used in network-A.

The input traffic for wireless FTP sessions for the first transport as illustrated in Fig. 3.a; the input traffic for wireless FTP sessions for the second transport (network-A) is as Fig. 3.b. The idle period (i.e. T2) after transmission in FTP is assumed to be substantially larger than the fade duration in the channel, thus T2 >> t2, (fading time has to be small otherwise the mobile device will close the connection). For wireline sessions, the average FTP transmission time is T1 whereas the average idle time per session is T2 (on/off model for FTP sessions); $\mathrm{T} 1$ and $\mathrm{T} 2$ are exponentially distributed.

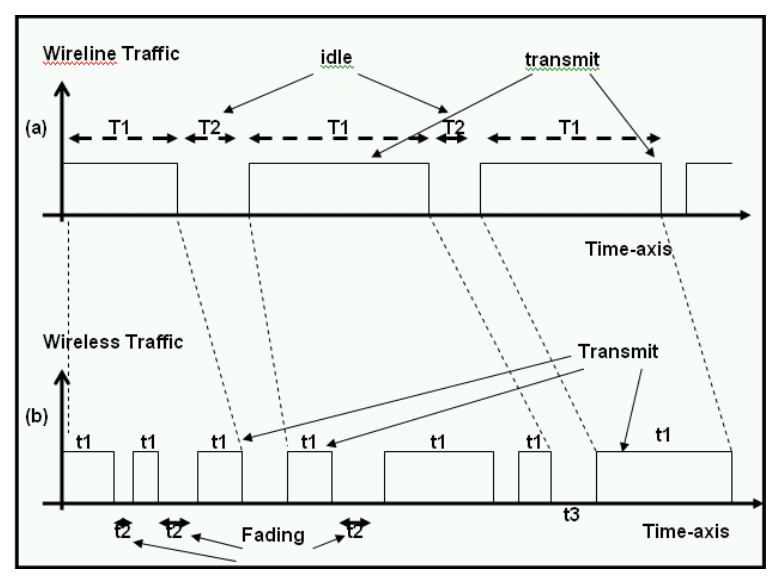

Fig. 3: (a) Shows the traffic model for wireline FTP

session. (b) Shows the traffic model for wireless FTP traffic after going through WLAN protocol transport.

Note that wireless FTP traffic between source "S-1" and wireless LAN (WLAN) follows similar distribution. For wireless FTP traffic leaving WLAN (here FTP traffic refers to FTP packets without errors due to RF link) and entering network-A., the following is true: let $\mathrm{t} 1$ be the average transmission time for wireless FTP sessions leaving the WLAN and entering network-A (note $\mathrm{t} 1$ and $\mathrm{T} 1$ are in general different due to the filtering process of I-TCP over the WLAN). 
However, T1=t1 when there is no fading over the wireless channel. Let $\mathrm{t} 2$ be the average fade duration for wireless traffic over the WLAN; $t 1$ and $t 2$ are exponentially distributed random variables (Jakes Model) ${ }^{[10]}$. Let $\mathrm{t} 3$ be the modified idle time, i.e. intertransmit time, for wireless traffic over network-A, and $N$ be a random variable representing the number of inter-fade periods during T1. Then, $N=T 1 / t 2$ and $T 1+$ $T 2=(N+1) x t 1+N t 2+t 3$.

Network Simulator NS (http://wwwmash.cs.berkeley.edu/ns/) was used as the basis for the simulation. Significant code modifications to the original simulation have been done to fit the purpose of this study. The test assumptions and parameters are listed: (1) A WLAN based on Carrier Sense Multiple Access/Collision Avoidance (CSMA/CA) was used for our LAN; concurrent sessions were established using TCP-Reno. (2) The LAN bandwidth is $10 \mathrm{Mbps}$, and the WLAN bandwidth is $11 \mathrm{Mbps}$, with a link delay of 10 microseconds. (3) Packet size (of 128 Bytes) means: TCP's payload. (4) Window size equals 32 packets. (5) The size of the FIFO queue was large enough to eliminate buffer overflows. The following parameters are set as: $T 1=50 \mathrm{sec} ; T 2=10 \mathrm{sec} ; \mathrm{t} 1=5 \mathrm{sec} ; t 2=1$ $\sec (t 2$ is the average fade duration over the airlink resulting from average bit error rate of $\left.10^{-1}\right)$; and $t 3=1$ sec.

\section{NUMERICAL RESULTS}

The following five cases were considered:

- Cases 1 and 2 were run without I-TCP:

○ In Case $1, \mathrm{U}=50, \mathrm{U} 1=25$, and $\mathrm{U} 2=25$, the bit error rate (BER) for the wireless sessions is set to $10^{-4}$. The throughput of network-A using our simulation is $790 \mathrm{kbps}$; approximately $7.9 \%$ utilization for network-A TCP/IP.

○ For Case 2, U=50, U1=25, and $\mathrm{U} 2=25$, the BER is changed $10^{-5}$. As a result, the throughput of network-A increased to 1497 kbps; $14.97 \%$ utilization of network-A.

- For Case 3 with $\mathrm{BER}=0$ (pure wireline TCP network with $\mathrm{U} 1=50$, the throughput increased to $2474 \mathrm{kbps}$. The above numbers indicate the significant degradation of TCP-Reno throughput performance due to RF packet losses.

- $\quad$ For Case 4, BER $=10^{-4}$, I-TCP was used with U1 = 25 and $\mathrm{U} 2=25$; the throughput recorded is 2433 kbps which is within $41 \mathrm{kbps}$ of Case 3. The degradation is due mainly to the additional randomness of wireless traffic Fig. . 3.b.

- For Case 5 with 50 wireless FTP sessions, (U2=50), and I-TCP (more randomness), the throughput has dropped further to $2385 \mathrm{kbps}$.

In summary, as the number of wireless FTP sessions increases, the overall throughput of the network-A decreases both with and without I-TCP. The degradation in throughput with I-TCP is minimal compared to a network without I-TCP. Under these simulation conditions, the maximum achievable throughput is within $89 \mathrm{kbps}$ from the throughput for pure wireline TCP network.

\section{CONCLUSIONS}

In this paper, the performance of the TCP over wireless links has been evaluated. Huge number of data applications on wireline networks use TCP/IP as an end-to-end flow and congestion avoidance protocol. TCP reacts to packets loss by retransmitting the missing packets and invoking congestion control mechanism. The use of the traditional TCP for wireless networks results in degraded end-to-end throughput and suboptimal performance. This study examined how to obtain the maximum achievable throughput for integrated wireline and wireless networks. Through simulation, it has already been noted that, as the packet loss due to fading is hidden from the wireline side by the I-TCP, which hides errors at the wireless side, a maximum throughput of $10 \%$ for wireless/wireline network is achievable.

The bottom line is that there is a need to adapt TCP protocol so that it can provide reliable data transfer at a maximum throughput possible for an integrated wireline and wireless networks. New or modified TCP protocols for wireless/wireline integrated networks should aim at achieving for exceeding this throughput.

\section{REFERENCES}

1. Bakre, A., and B. Badrinath, 1995. "I-TCP: Indirect TCP for mobile hosts”, in. $15^{\text {th }}$ Int. Conf. on Distributed Computing System.

2. Balakrishnan, H., S. Seshan, and R. Katz, 1995. "Improving Reliable Transport and Handoff Performance in Cellular Wireless Networks". ACM Wireless Networks, Vol 1, No 4.

3. Balakrishnan, H., V. Padmanabhan, S. Seshan, and R. Katz, 1997. "A Comparison of Mechanisms for Improving TCP Performance over Wireless Links", IEEE/ACM Transactions on Networking, Vol. 5, No. 6.

4. Brakmo, L., S. O’Malley, and L. Peterson, 1994. "TCP Vegas: New Techniques for Congestion Detection and Avoidance", in Proc. ACM SIGCOMM.

5. Caceres, R., P. Dauzig , S. Jamin, and D. Mitzel, 1991, "Characteristics of Wide Area TCP/IP Conversation", in Proc. ACM SIGCOMM, August, pp 101-112.

6. Chaskar, H., T. Lakshman., and U. Madhow, 1996. "The Design of Interfaces For TCP/IP Over Wireless", in Proc. IEEE Milcom.

7. Comer, D.E., 1991. "Interworking with TCP/IP", Vol 1, Prentice-Hall Inc. 
8. Crow, B., I. Widjaja, J. Kim, and P. Sakai, 1997. "IEEE 802.11 Wireless Local Area Networks", IEEE Communications Magazine.

9. Jacobson, V., 1998. "Congestion Avoidance and Control", in Proc. Of SIGCOMM.

10. Jakes, W., 1993. "Microwave Mobile Communications", reissued by IEEE Press.

11. Lakshman, T., and U. Madhow, 1997. "The Performance of TCP/IP for Networks with High Bandwidth-Delay Products and Random Loss". IEEE/ACM Trans. on Networking, Vol. 5, No. 3.

12. Paxon, V., 1994. "Empirically Derived Analytic Models of Wide-Area TCP Connections", IEEE/ACM Transactions on Networking, Vol. 2, No 4.

13. Qaddoura, E., R. Prakash, and L. Tamil, 2000. "Aggressive Error Recover For TCP Over Wireless Links", Integrated Computer-Aided Engineering Journal, a special issue on Distributed Computing and Networking.

14. Ramakrishnan, K., and R. Jain, 1998. “A Binary Feedback Scheme for Congestion Avoidance in Computer Networks with Connectionless Network Layer". In Proc. ACM SIGCOMM.

15. Stevens, W.R., 1994. "TCP/IP Illustrated, Volume 1, The Protocols". Addison Wesley Longman, Inc., November.

16. RFC 793, 1981. "Transmission Control Protocol", DARPA Internet Program, Protocol Specification, September.
17. Brown, K. and S. Singh, 1996. "M-TCP: TCP for Mobile Cellular Networks", INFOCOM96.

18. Natani, A., Jakilinki, J., M. Mahsin, and V. Sharma, 2001. "TCP for Wireless Networks". Computer Science Program. Univ. of Texas at Dallas, Richardson, TX 75083-0688.

19. Chiasserini, C., F. and M. Meo, 2001. "Improving TCP over Wireless Through adaptive Link Layer Setting". In IEEE GLOBECOM, Symposium on Internet Performance.

20. $\mathrm{Xu} \mathrm{S}$. and T. Saadawi, 2001. "Does the IEEE 802.11 MAC protocol work well in multi-hop wireless ad-hoc networks?", IEEE communication Magazine, Vol 39, No. 6.

21. Jiang, R., V. Gupta, and C. Ravishankar, 2001. "Interactions between TCP and the IEEE 802.11 MAC Protocol". IEEE Communication Magazine.

22. Peterson, L., and Davie L., B.S., 2003. "Computer Networks: A System Approach" $3^{\text {rd }}$ edition, Morgan Kaufmann.

23. Paxson, V. 1999. "End-to-end internet packet dynamics". IEEEIACM Trans. Networking 277292. 